Journal of Computer Science 8 (7): 1177-1183, 2012

ISSN 1549-3636

(C) 2012 Science Publications

\title{
An Optimized Ad Hoc on Demand Distance Vector Routing Protocol for Wireless Networks
}

\author{
${ }^{1}$ V. Ponniyin Selvan and ${ }^{2}$ S. Senthil Kumar \\ ${ }^{1}$ Department of ECE, Anna University of Technology, Coimbatore, TamilNadu, India \\ ${ }^{2}$ Department of EEE, Govt College Engineering, Salem, TamilNadu, India
}

\begin{abstract}
Problem statement: Wireless Mesh Networks (WMN) is communication network made up of mesh routers and mesh clients, with mesh routers forming the backbone of the network. The WMN can be accessed by other networks through gateways and bridging functions in the mesh routers. The mesh clients can either be static or mobile with an option of forming a client mesh network with the mesh routers. Routing in WMN is through multi-hop relays including the access points and gateways. Many ad hoc routing protocols such as Highly Dynamic Destination-Sequenced Distance Vector (DSDV), Ad Hoc on Demand Distance Vector (AODV) are used in WMN. Though these routing protocols are used in WMN, the protocols do not address constraints inherent to WSN, due to which network resources are not properly utilized and there is a fall in Quality of Service (QoS). Thus, these routing protocols are enhanced with new routing metrics more appropriate to WMN. Approach: In this study, a Modified on-demand routing algorithm for Mobile Ad-hoc Networks (MANETs), Ant Mesh Network AODV is proposed. AODV is modified to include the ant colony based optimization. The modified routing protocol improves the throughput and decreases the packet loss along with reduction in routing overhead. Results and Conclusion: The proposed optimization technique decreases the energy overhead of nodes in the network which are one hop neighbor to the sink.
\end{abstract}

Key words: Wireless Mesh Networks (WMN), Ad Hoc On Demand Distance Vector (AODV), Quality of Service (QoS), Ant Colony Optimization (ACO)

\section{INTRODUCTION}

A wireless network is a collection of wireless nodes communicating with each other without any base infrastructure. Routes for forwarding data packets from source to destination nodes are dynamically established through multi hop (Macker and Corson, 2003). Wireless routing protocols can be broadly classified into pro active routing protocols and reactive routing protocols. In the proactive routing protocol, routing tables are maintained with discovering routes as the network is formed and are constantly updated to accommodate the dynamic nature of the wireless network. Distance Sequence Distance Vector (DSDV) (Perkins and Bhagwat, 1994) routing protocol, Optimized Link State Routing protocol (OLSR) (Clausen and Jaquet, 2003) is most commonly used proactive routing protocol. In reactive routing protocols, routes are discovered from source to destination only when data packets need to be transmitted. Popular reactive routing protocols include Ad hoc on demand Distance Vector (AODV) routing protocol (Anuradha and Dutta, 2010), Dynamic Source Routing (DSR) protocol (David et al., 2001).
Wireless Mesh Networks (WMN) (Yuan et al., 2005) is communication network made up of mesh routers and mesh clients, with mesh routers forming the backbone of the network. The WMN can be accessed by other networks through gateways and bridging functions in the mesh routers (Jayakumar, 2008). The $\mathrm{WMN}$ is generally composed of the infrastructure component and many ad hoc networks. The main advantages of WMN are its low up-front cost, easy deployment, easy maintenance, robustness and reliable coverage. Similar to a wireless network, all the nodes act as routers and automatically establish a mesh connection. The mesh clients can either be static or mobile with an option of forming a client mesh network with the mesh routers. Routing in WMN is through multi-hop relays including the access points and gateways. Many ad hoc routing protocols such as DSDV, AODV are used in WMN. Though these routing protocols are used in WMN, the protocols do not address constraints inherent to WSN, due to which network resources are not properly utilized and there is a fall in Quality of Service (QoS). Thus, these routing protocols are enhanced with new routing metrics more appropriate to $\mathrm{WMN}$. 


\section{J. Computer Sci., 8 (7): 1177-1183, 2012}

WMN are self-forming, self-organized and selfconfigured multi-hop wireless networks. It enhances the network performance due to its mesh connectivity and flexible network architecture. The network can be extended gradually as required. WMNs support both Internet and peer-to-peer communications and can be integrated with other wireless networks (Jun and Sichitiu, 2003). The mesh routers do not face power consumption constraints as in a wireless network. Routing and configuration functions are mainly taken up by the mesh routers. The main issues faced by WMNs are scalable, network integration and security (Maalla et al., 2009).

In this study it is proposed to implement a modified AODV using the concepts of Ant Colony Optimization to improve the routes taken by the data in a multi hop wireless mesh network.

Related works: Tingrui et al. (2009) proposed an Improved Hierarchical AODV routing protocol (IHAODV). The proposed protocol incurs less routing overhead for finding alternate routes when a route is lost, thus improving the overall performance in the network. It also has better scalability. A novel technique for IH-AODV term fresh route detection is presented. NS-2 simulation was used to compare both AODV and IH-AODV. Simulation results show that IH-AODV performs better than AODV with better metrics in large hybrid WMNs.

Yun-Qing et al. (2007) proposed MODVWLS an improved AODV by using weighted path as routing metric. In the proposed method, the weight of each hop is computed using the node's bandwidth, buffer queue and throughput. The route with minimum weight is used as the preferred route. Methods for weight calculation, message formats, route discovering and maintenance process are discussed. To evaluate the performance of the proposed protocol, several simulations are conducted using ns-2. Simulation results show MODVWLS is better than AODV on packet delivery fraction, the average end-to-end delay and normalized routing load, so it is more suitable for wireless network.

Guo-Ping and Jian-Hui (2010) proposed AODV_D an improved AODV, for better delivery of data packets. WMN using AODV selects routes with the least hops for data packet delivery without considering the path stability. When the nodes are highly mobile, the routes have shorter lifetime. Thus, stability of the route is less and routing overheads are increased due to route recovery. The proposed method uses a routing table to select the less hops but a high stability path to send data packets. The AODV_D routing protocol is activated by static nodes, sports node has the option to activate or not activate this routing mechanism. NS-2 simulation results show that the delivery rate of data packets increased with a reduction in the average end to end delay and packetloss-rate in the proposed AODV_D routing protocol.
Imani et al. (2011) presented AODV-Mesh, for the WMNs. This protocol is an extension of the AODV routing protocol enhanced to the specifications of the WMNs. The proposed method introduces two route recovery mechanisms. AODV-Mesh uses the local repair mechanism and AODV-Mesh NoLR does not use the local repair mechanism. Simulation results show that not using the local repair mechanism in the AODV-Mesh algorithm improves the performance and efficiency.

Ajmal et al. (2010) presented a new hybrid routing metric to enhance AODV for routing in WMNs. The proposed method selects the path for routing based on load balancing, link quality, successful transmission rate and number of hops. The experimental results show that the path selected using the proposed method is more optimal than the standard AODV.

Li et al. (2010) proposed a Security Enhanced AODV routing protocol (SEAODV) for WMN. The proposed method uses Pairwise Transient Key (PTK) and Group Transient Key (GTK) to authenticate unicast and broadcast routing messages respectively. The PTK and GTK ensure secure route discovery process between two nodes. Blom's key pre-distribution scheme is utilized for computing the PTK through the flooding of enhanced HELLO message. GTK is distributed after establishing PTK, a unique PTK is shared by each pair of nodes, whereas GTK is shared between the node and all its one-hop neighbors. The routing message authenticity and integrity is assured in hop-by-hop by the use of Message Authentication Code (MAC) attached to the routing message. Simulation results show that the proposed SEAODV outperforms ARAN and SAODV in terms of computation cost and route acquisition latency. The proposed protocol SEAODV is also more effective in preventing identified routing attacks.

\section{MATERIALS AND METHODS}

An Ant Colony Optimization algorithm (ACO) is based on ant's behavioral mechanisms such as cooperation and adapting (Ali et al., 2010). Metaheuristics are formed to solve optimization problems. ACO algorithms are based on the following ideas (Dorigo et al., 1999):

- Solution for a problem is obtained by the path followed by an ant

- The quantity of pheromone left on the path by ants represents the quality of the solution for the problem

- When an ant has to choose between two or more paths, paths with higher pheromone is generally chosen

- In due course, ants converge to a short path, which would represent the optimum or a near-optimum solution for the target problem 


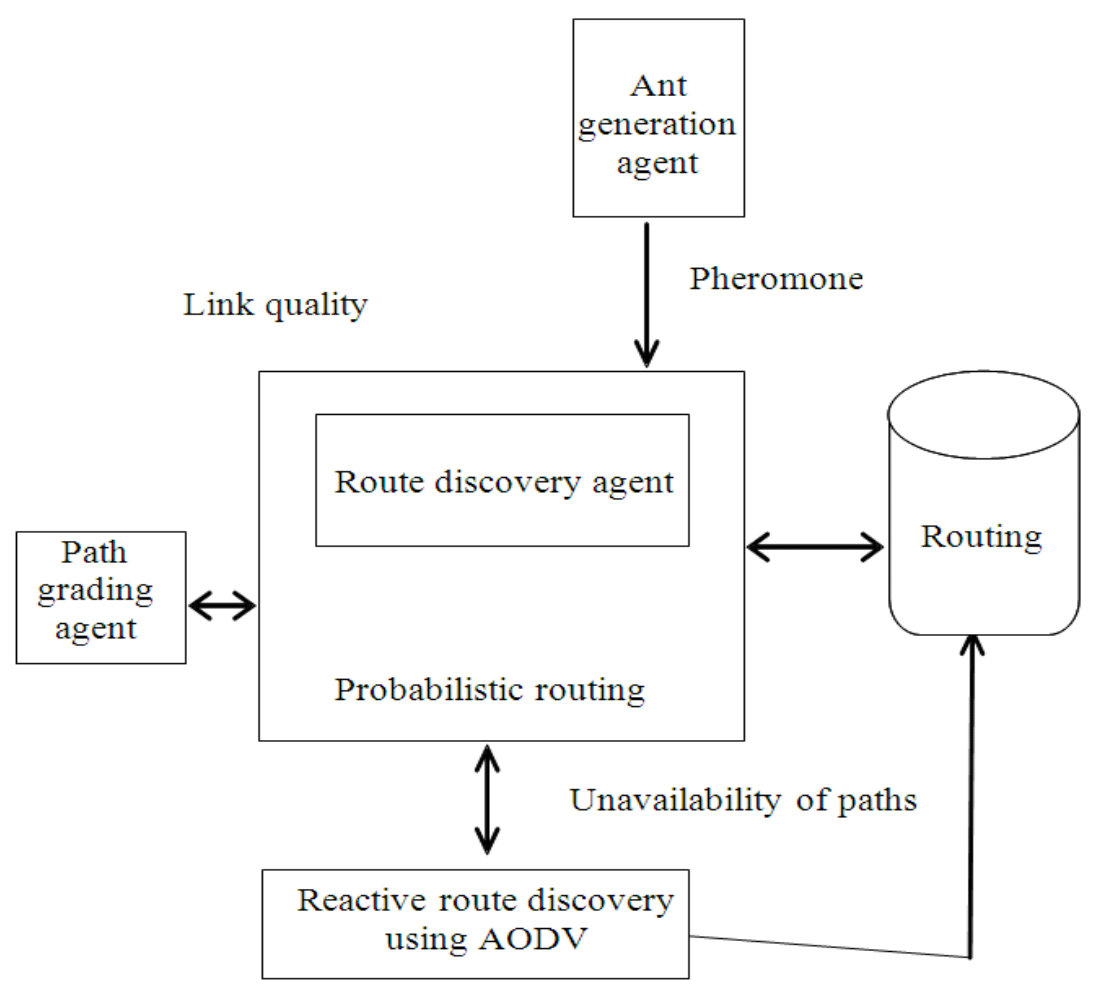

Fig. 1: The proposed routing algorithm

The design of an ACO algorithm involves the following (Bonabeau et al., 1999):

- The problem has to be appropriately represented. The ants should be able to incrementally construct and modify according to probabilistic transition rule. It is based on the amount of pheromone on the trail and on a local, problem-dependent heuristic

- Constraints which help model valid solutions

- An objective function (h) to measure the quality of components included in the partial solution during construction of the solution

- Modifying pheromone trail (t) according to the rule

- The solution is constructed in steps using a probabilistic transition rule. The probabilistic transition rule found in the value of the heuristic function $(\mathrm{h})$ and pheromone trail $(\mathrm{t})$

Artificial ants have several characteristics similar to real ants. Artificial ants give preference for paths with a larger amount of pheromone. Shorter paths have larger growth in amount of pheromone.

At each iteration, the pheromone values are updated by all them ants that have built a solution in the iteration itself. The pheromone $\tau_{\mathrm{ij}}$, associated with the edge joining nodes $i$ and $j$, is updated as follows:

$$
\tau_{\mathrm{ij}} \leftarrow(1-\rho) \cdot \tau_{\mathrm{ij}}+\sum_{\mathrm{k}=1}^{\mathrm{m}} \Delta \tau_{\mathrm{ij}}^{\mathrm{k}}
$$

where, $\rho$ is the evaporation rate, $m$ is the number of ants and $\Delta \tau_{\mathrm{ij}}^{\mathrm{k}}$ is the quantity of pheromone laid on edge $(\mathrm{i}, \mathrm{j})$ by ant $\mathrm{k}$ :

$$
\Delta \tau_{\mathrm{ij}}^{\mathrm{k}}=\left\{\begin{array}{c}
\mathrm{Q} / \mathrm{L}_{\mathrm{k}} \\
0
\end{array}\right.
$$

where, $\mathrm{Q}$ is a constant and $\mathrm{L}_{\mathrm{k}}$ is the length of the path constructed by ant $\mathrm{k}$. In the construction of a solution, ants select the following node to be visited through a stochastic mechanism. The partial solution is constructed by:

$$
\mathrm{p}\left(\mathrm{c}_{\mathrm{ij}} \mid \mathrm{s}^{\mathrm{p}}\right)=\frac{\tau_{\mathrm{ij}}^{\alpha} \eta_{\mathrm{ij}}^{\beta}}{\sum \mathrm{c}_{\mathrm{ij}} \in \mathrm{N}\left(\mathrm{s}^{\mathrm{p}}\right) \tau_{\mathrm{ij}}^{\alpha} \eta_{\mathrm{ij}}^{\beta}}{ }^{\prime} \forall \mathrm{c}_{\mathrm{ij}} \in \mathrm{N}\left(\mathrm{s}^{\mathrm{p}}\right)
$$

where, $\mathrm{N}\left(\mathrm{s}^{\mathrm{p}}\right)$ is the set of feasible components

The proposed ACO based proactive routing process is shown in Fig. 1. In the proposed routing protocol Load Balanced Ants- ADOV routing protocol, every node maintains a pheromone table and a probability routing table. 


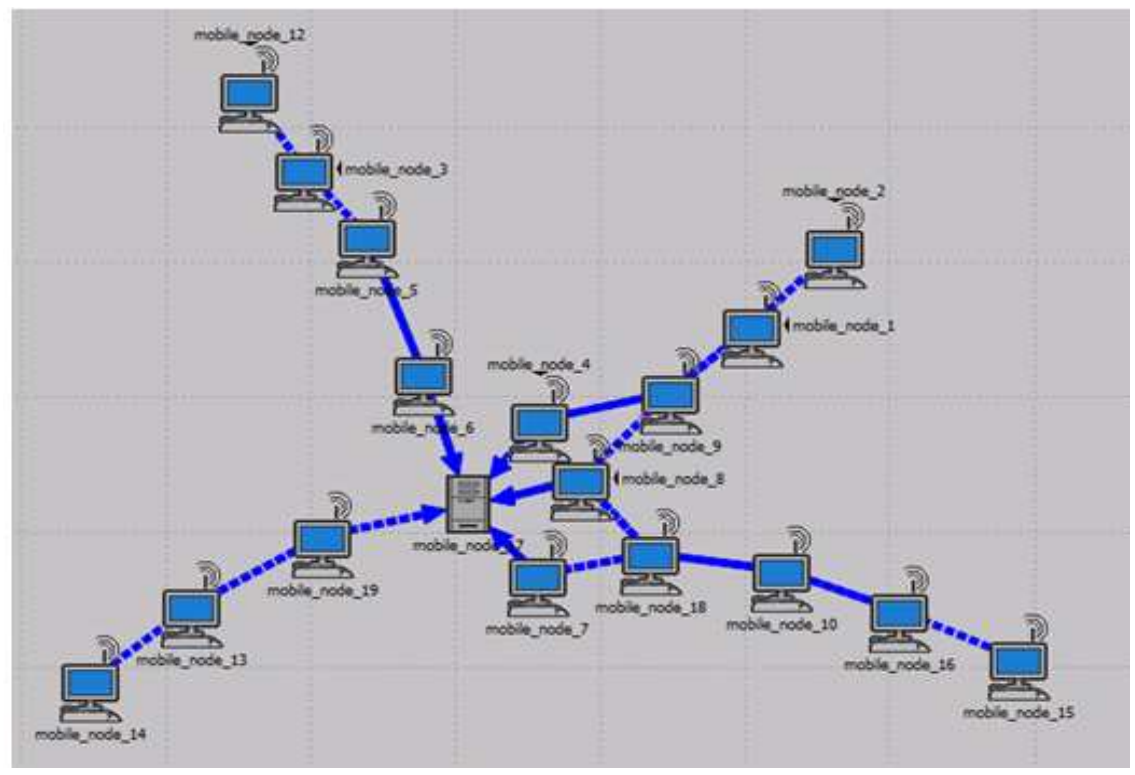

Fig. 2: The routes taken in the WMN using AODV routing protocol

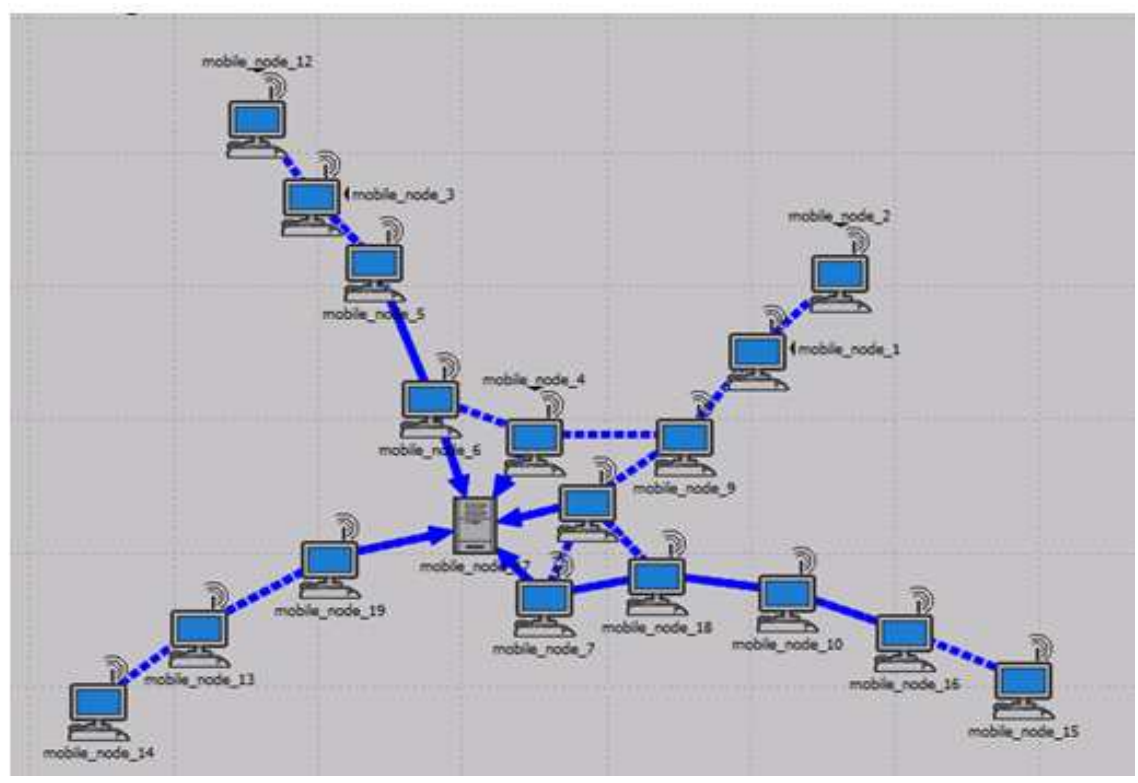

Fig. 3: The routes taken in the WMN using the proposed routing protocol

Pheromone values are maintained in all the nodes of the network for each destination-neighbor pairs. The pheromone values for all neighbors for a particular destination are initialized to the same value to make the search unbiased. These initial values of the pheromones change with respect to the number of ants moving towards a particular neighbor. The path in which more ants move towards a link is the optimal path to the destination. The pheromone entry raises as more ants move and more probability is assigned to the neighbor. The probability value of the neighbor is also affected by the link life, processing power of the neighbors and energy depletion rate of the neighbors.

Information about the quality of the nodes such as battery's energy and processing power is collected as request ants pass through the nodes. Normalized index with value varying from $0-1$ is used to express the information. The quality of the overall path is the 
product of the normalized index value of all the nodes. The destination node on receiving the information from the request ants checks it against the reference value it maintains. The pheromone values at the intermediate nodes are updated as destination node sends out reply ants with the grades. The pheromones deposited reduces according to the evaporation rate, this enables the nodes to forget old paths as topology of the wireless network changes.

\section{RESULTS AND DISCUSSION}

The experimental setup consisted of a single sink with 17 nodes. The maximum number of hops to reach the sink is 4 . Each node has a transmission power of $0.03 \mathrm{~W}$ and maximum Bandwidth of $1 \mathrm{MW}$. Two experiments were conducted with the first experiment running on AODV routing protocol and the second experiment using the proposed LBA-AODV routing protocol. Figure 2-3 show the snapshot of route created by the AODV routing protocol and the proposed protocol.

From Fig. 3 it can be seen that the load balancing at the one hop node is much better compared to AODV routing protocol which not only improves the battery life of the one hop node, but also improves the load balancing.

Figure 4 shows the route discovery time. The proposed routing protocol (blue graph) takes much longer for route discovery compared to AODV due to the ant technique. However being a fixed node the WMN routes do not change continuously and hence the delay be justified.

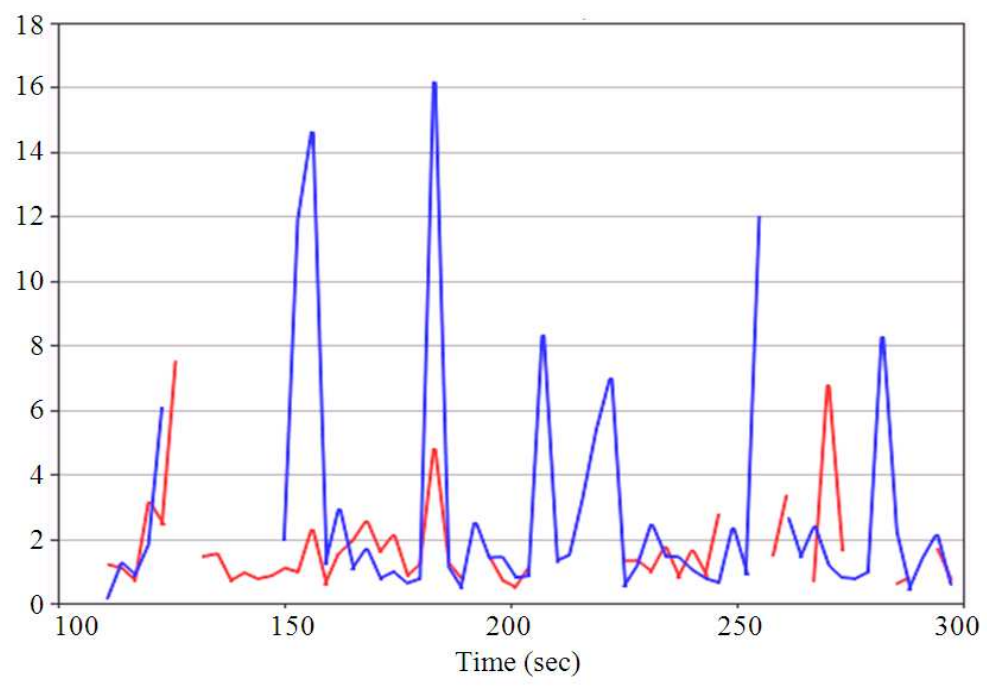

Fig. 4: Route discovery time of the proposed Protocol compared with AODV

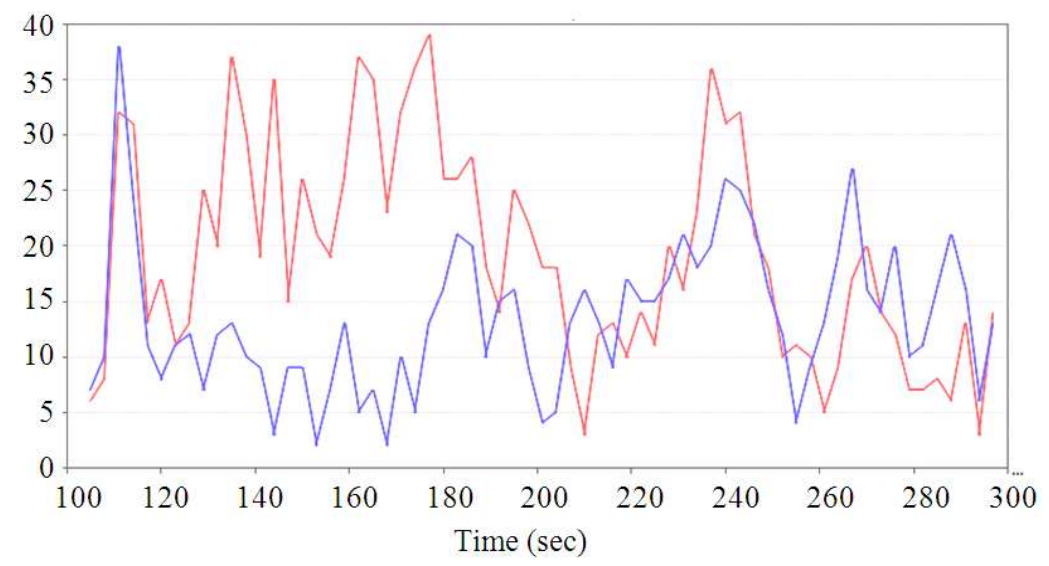

Fig. 5: Routing traffic received of the proposed protocol compared with AODV 
J. Computer Sci., 8 (7): 1177-1183, 2012

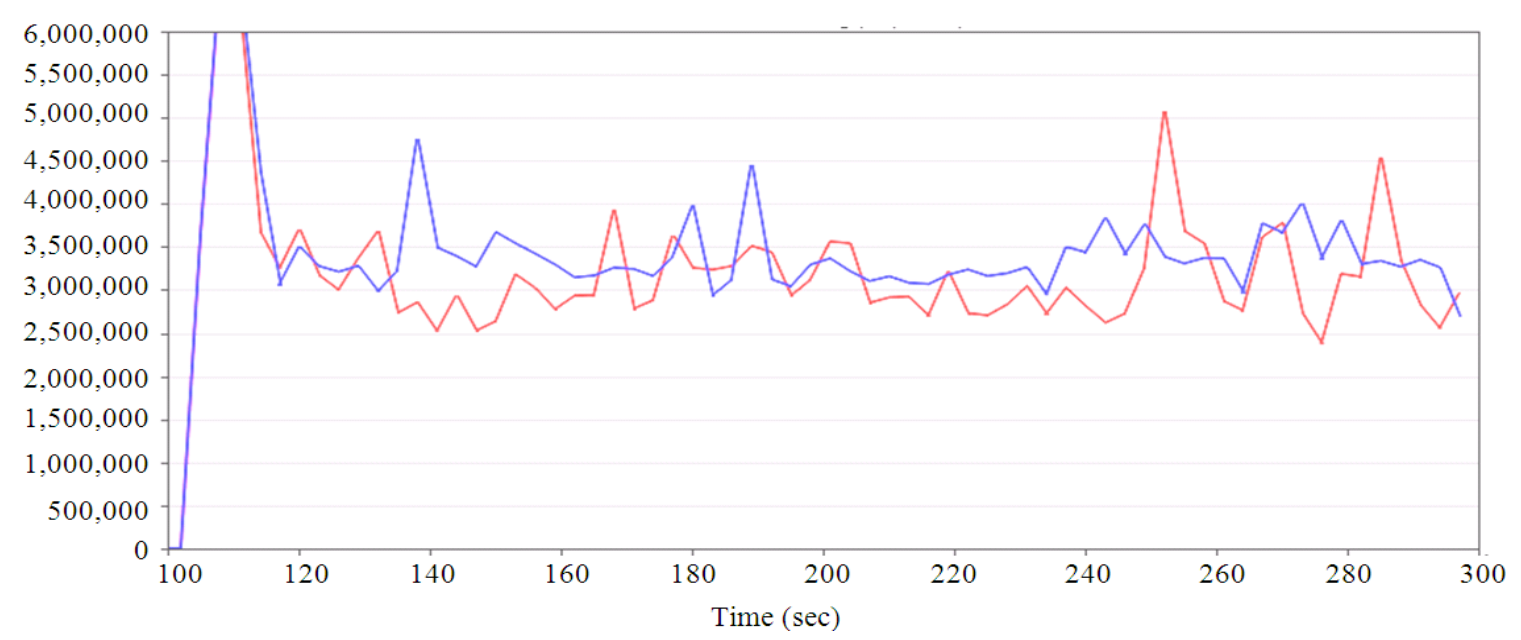

Fig. 6: Throughput of the proposed system

Figure 5 and 6 shows the overall routing traffic received by the nodes in the network. It can be seen that the proposed method reduces the control packet overheads which is advantageous in bandwidth constrained environment. Similarly the throughput of the proposed system improves marginally compared to existing AODV.

\section{CONCLUSION}

In this study a novel routing protocol which is an enhancement of the existing AODV routing protocol to address the deficiencies of WMN was proposed. The proposed protocol was based on Ant Colony Optimization (ACO) where in based on the link quality and the load, the ants forage using newer routes so that delay is avoided. The proposed method improves the load balancing at the one hop nodes which not only avoids congestion but also improves the battery life of the one hop nodes which is crucial for sensor nodes connected in mesh format.

\section{REFERENCES}

Ajmal, M.M., K. Mahmood and S.A. Madani, 2010. Efficient routing in wireless mesh network by enhanced AODV. Proceeding of the International Conference on Information and Emerging Technologies, Jun. 14-16, IEEE Xplore Press, Karachi, pp: $1-7 . \quad$ DOI: 10.1109/ICIET.2010.5625706

Ali, A., M.A. Belal and M.B. Al-Zoubi, 2010. Load balancing of distributed systems based on multiple ant colony optimization. Am. J. Applied Sci., 7: 428-433. DOI: 10.3844/ajassp.2010.428.433
Anuradha, B. and P. Dutta, 2010. Link stability and node energy conscious local route-repair scheme for mobile ad hoc networks. Am. J. Applied Sci., 7: 1139-1147. DOI: 10.3844/ajassp. 2010.1139.1147

Bonabeau, E., M. Dorigo and G. Theraulaz, 1999. Swarm Intelligence: From Natural to Artificial Systems. 1st Edn., Oxford University Press, New York, ISBN-10: 0195131592, pp: 307.

Clausen, T. and P. Jaquet, 2003. Optimized link state routing protocol. The Internet Society.

David, B., J. David, A. Maltz and J. Broch, 2000. The dynamic source routing protocol for multihop wireless ad hoc networks. Ad Hoc Netw.

Dorigo, M., G. Di Caro and L.M. Gambardella, 1999. Ant algorithms for discrete optimization. Artificial Life, 5: 137-172. DOI: 10.1162/106454699568728

Guo-Ping, X. and L. Jian-Hui, 2010. Improvement of AODV routing protocol based on wireless mesh networks. Proceedings of the Computer, Mechatronics, Control and Electronic Engineering (CMCE), International Conference, Aug. 24-26, IEEE Xplore Press, Changchun, pp: 372-375. DOI: 10.1109/CMCE.2010.5610515

Imani, M., B. Hassanabadi and M. Naderi, 2011. Refinement of AODV routing algorithm for Wireless Mesh Networks (WMNs). Proceedings of the 19th Iranian Conference on Electrical Engineering, May 17-19, IEEE Xplore Press, Tehran, Iran, pp: 1-1.

Jayakumar, G., 2008. Performance comparison of two on-demand routing protocols for ad-hoc networks based on the random way point mobility model. Am. J. Applied Sci., 5: 659-664. DOI: 10.3844/ajassp.2008.659.664 
Jun, J. and M.L. Sichitiu, 2003. The nominal capacity of wireless mesh networks. IEEE Wireless Commun., 10: 8-14. DOI: 10.1109/MWC.2003.1241089

Li, C., Z. Wang and C. Yang, 2010. SEAODV: A Security Enhanced AODV routing protocol for wireless mesh networks. Trans. Comput. Sci., 6480: 1-16.

Maalla, A., C. Wei and H.J. Taha, 2009. Optimal power multicast problem in wireless mesh networks by using a hybrid particle swarm optimization. Am. J. Applied Sci., 6: 1758-1762. DOI: 10.3844/ajassp.2009.1758.1762

Macker, J.P. and S. Corson, 2003. Mobile ad hoc networks (MANET): Routing technology for Dynamic, Wireless Networking". In: Ad Hoc Networking, Basagni, S., M. Conti, S. Giordano and I. Stojmenovic, (Eds.). IEEE Press Wiley, New York, pp: 255-274.

Perkins, C.E. and P. Bhagwat, 1994. Highly dynamic destination-sequenced distance-vector routing (dsdv) for mobile computers. ACM SIGCOMM Comput. Commun. Rev., 24: 234-244. DOI: $10.1145 / 190314.190336$
Tingrui, P., Z. Wenli, Z. Zhaoxiao and P. Tao, 2009. An improved hierarchical aodv routing protocol for hybrid wireless mesh network. Proceedings of the International Conference on Networks Security, Apr. 25-26, IEEE Xplore Press, Xiangtan, pp: 588593. DOI: 10.1109/NSWCTC.2009.112

Yuan, Y., Z. Yang and J. He, 2005. An adaptive modulation scaling scheme for quality of services ensurance in wireless sensor networks. Am. J. Applied Sci., 2: 734-738. DOI: 10.3844/ajassp.2005.734.738

Yun-Qing, F., W. Song-Jian and Z. Ming-Yang, 2007. Research on weighted link based routing protocol for wireless mesh network. Proceedinds of the IET Conference on Wireless, Mobile and Sensor Networks, Dec. 12-14, IEEE Xplore Press, China, pp: 293-296. 